\title{
Fifth European Conference on Controlled Fusion and Plasma Physics
}

\author{
Grenoble, France, 21-25 August 1972
}

The European Conferences on Controlled Fusion and Plasma Physics have become traditional by now. These Conferences developed from meetings of the European Study Group on Fusion Problems, established by CERN, and have rapidly enlarged to a truly European basis, in spite of the fact that, in the beginning, they were borne only by self-appointed committees. The First Conference was held in Munich back in 1966. Further Conferences followed at Stockholm in 1967, at Utrecht in 1969, and at Rome in 1970. The latter was the first meeting of the European plasma physicists to be organised by the European Physical Society through its Plasma Physics Division.

The Fifth European Conference on Controlled Fusion and Plasma Physics was held at Grenoble (France). About 350 plasma physicists participated in the meeting. Noteworthy was the strong interest the Conference found outside Europe : close to $20 \%$ of the participants came from countries not belonging to the area of the European Physical Society, in particular from the United States, but also from Australia, Canada, Egypt, and Japan.

At the meeting 20 invited lectures and about 170 contributed papers were given. The selection of the papers and their grouping into sessions was in the hands of an International Paper Selection and Programme Committee, consisting of $\mathrm{G}$. Laval (France), B. Lehnert (Sweden), D.R. Sweetman (UK) and H. Zwicker (German Dem. Rep.). The invited lectures were given in plenary sessions, held in the morning, whereas the contributed papers were presented in four parallel sessions in the afternoon. A visit to the Grenoble Plasma Physics laboratories (Service d'lonique Générale of the Département de Physique du Plasma et de la Fusion Contrôlée of the Commissariat à l'Energie Atomique) was also included in the programme.

The invited papers were concerned with the following topics:

\section{Experiments on the ST Tokamak}

(W. Stodiek, Princeton)

Experiments on USSR Tokamaks

(V.L. Strelkov, Moscow)

Neoclassical Diffusion in a Toroidal Octupole (T. Ohkawa, San Diego)
Survey of Scyllac Experiments

(F.C. Jahoda, Los Alamos)

High- $\beta$ Toroidal Experiments

(D.C. Robinson, Culham)

Toroidal Screw Pinches with Non-Circular Cross Section

(H. Zwicker, Garching)

Confinement Studies in $2 \mathrm{X}-11$

(F.L. Coensgen, Livermore)

Anomalous Resistivity

(R.Z. Sagdeev, Moscow)

Computer Simulation of Collisionless Plasmas (D. Biskamp, Garching)

Plasma Diffusion in Two and Three Dimensions (J.B. Taylor, Culham)

Results on Shock Properties (E. Hintz, Julich)

Containment and RF Heating Studies on the Proto-Cleo Stellarator

(D.J. Lees and W. Millar, Culham)

Numerical Models of Plasma

Evolution in Tokamak Devices

(C. Mercier, Fontenay-aux-Roses)

Laser Plasmas and Controlled Fusion

(J.L. Bobin, Limeil)

Progress on Plasma Focus

(Ch. Maisonnier, Frascati)

Problems in the Physics of

Plasma-Laser Interaction

(M.N. Rosenbluth, Princeton)

Comments on Pulsed Thermonuclear Systems

(E.P. Velikhov, Moscow)

The Activity of the European Community on Controlled Fusion

(D. Palumbo, Brussels)

Research and Prospects on Controlled Thermonuclear Fusion in the USA.

(R.W. Gould, Washington)

Research and Prospects on Controlled Thermonuclear Fusion in the USSR.

(L.A. Artsimovich, Moscow;

read by B.B. Kadomtsev)

Considerable progress was reported in all fields. Most of the effort in the research, in view of the realization of controlled fusion, continues to be made along the line of magnetic plasma confinement in toroidal geometry, and in particular on Tokamak

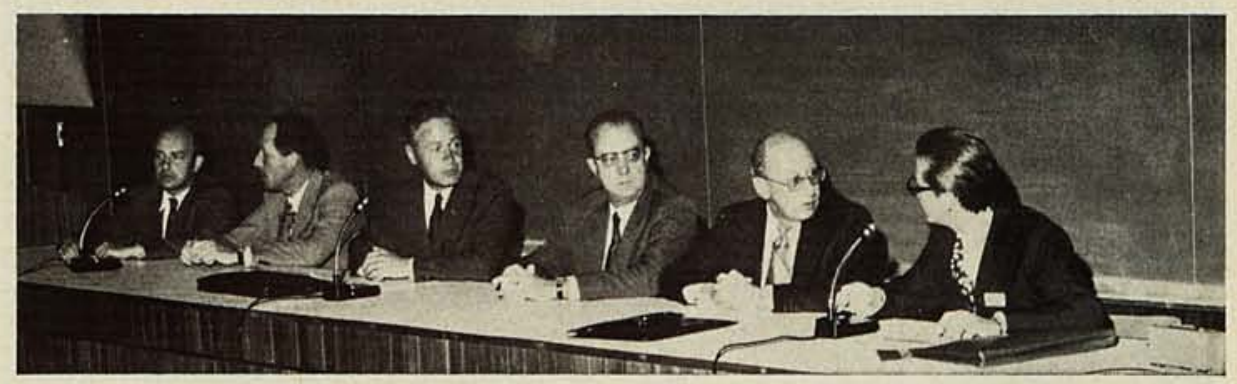

At the Fifth European Conference on Controlled Fusion and Plasma Physics (from left to right): B. Lehnert; the Mayor of Grenoble; M. Trocheris; the Representative of the Prefect of Iserre; M. Pascal; and T. Consoli. devices. New details about the behaviour of these latter machines have improved their physical understanding and further progress has been made as far as their performance is concerned. Electron temperatures of $3 \mathrm{keV}$ have been reached at densities of about $10^{13}$ particles per $\mathrm{cm}^{3}$, whereas the ion temperature is smaller by a factor of 4 . The confinement-times range somewhat above $10^{-2} \mathrm{~s}$, and seem to increase with temperature. So far, no fundamental difficulty has been encountered in these devices which would indicate that their performance cannot be further improved by going to higher currents. More work is, however, necessary to understand in detail the mechanisms which govern the losses in these and other machines. So far, it is clear that these losses must not be anomalous in the sense of being 'Bohm-like', but, on the other hand, it seems that there is generally an enhancement over the purely collisional value. Appreciable progress has also been made in the development of plasma heating methods (RF heating, turbulent heating, injection of energetic particles) to supplement or substitute ohmic heating which is likely to be insufficient for arriving at the ignition temperature of fusion heating. Their practical application on toroidal devices is, however, only in a preliminary stage. In this context, the observation of an increase in plasma resistivity and, hence, an enhancement of ohmic heating when RF waves are injected into the plasma, is noteworthy.

Also in the field of plasmas at higher kinetic pressure, produced by pinching, the emphasis of the work is on toroidal systems. Interesting new prospects are opened up by inves- 
tigations about systems with non-circular cross-section.

A considerably increased effort on pulsed systems was noted. First, the generation of dense and hot plasma by intense laser beams may be mentioned here, where the possibility of using the laser light also for compressing and partially confining the plasma is now under discussion. In parallel, work is being pursued on the use of relativistic electron beams and rapid compression. In the field of plasma focus, the use of refined diagnostics has led to a better understanding of the underlying physics.

New results were reported on the plasma behaviour in mirror machines and on plasma instabilities and turbulence in general. In the latter field, a combined effort, experimental, nume- rical and computational, is being made now to reach a better understanding of the nonlinear phenomena involved and the related transport properties of the plasma. The results reported indicate that weak turbulence theory, whereas it provides good qualitative insight, is quantitatively unsatisfactory even at fairly low turbulence levels.

The success of the Conference was largely due to T. Consoli and E. Canobbio, and the Grenoble Organizing Committee, who prepared the meeting excellently and ran it smoothly. Financial help by the Commissariat à l'Energie Atomique, Paris, and the Commission of the European Communities, Brussels, is gratefully acknowledged.

F. Engelmann

\section{Selected Problems in Magnetism}

\section{Bochum, Fed. Rep. of Germany, 25-28 July 1972}

The Magnetism Section of the Condensed Matter Division of EPS held its inaugural meeting in Bochum, 25-28 July 1972. There were about 200 participants from 17 European and some non-European countries.

The topics and details of the programme were determined by an international programme committee comprising J. Als-Nielsen (Denmark), G. Heber (German Dem. Rep.), S. Krupicka (CSSR), S. Methfessel (Fed. Rep. of Germany), A.R. Miedema ( $\mathrm{Ne}$ therlands), S. Shtrikman (Israel), D. Wagner (Fed. Rep. of Germany), and E.P. Wohlfarth (U.K.). The decisions on topics and speakers to be invited were made after a call for papers which was submitted to the national representatives of the Magnetism Section. In this way, it was hoped, a survey of the current activities in Europe could be given and presented at the conference. Out of a large number of proposals, the following were chosen as topics of the conference as being of the greatest general interest :

Critical phenomena,

Hubbard model,

Itinerant magnetism,

Localized moments,

Magnetic resonance,

Magneto-optics,

Rare earths,

Symmetries and structures.
The sessions of the meeting were subdivided into general sessions, which were held in the morning, and workshop sessions, which were held in the afternoon. The general sessions contained talks mostly surveying a specific field which was connected with the subject of a workshop session. There were three parallel workshop sessions every day, each session devoted to a different topic. In these sessions, there was ample time for discussions or short communications by participants, and sometimes heated discussions on controversial subjets took place.

For those participating, it was a particular pleasure to welcome L.F. Bates (Nottingham) and E.I. Kondorsky (Moscow) who attended the conference as guests of honour and who delivered talks on 'A link between past and present in European magnetism' and 'On the band theory of magnetic anisotropy of $\mathrm{Ni}$ and dilute $\mathrm{Ni}$ alloys', respectively.

As an inaugural meeting of a Section of an EPS Specialized Division the conference seems to have succeeded admirably in bringing together Europeans practising one of the oldest of the physical sciences, which is today perhaps more topical than ever. The Section is grateful to all those who helped to make this conference the success it was.

E.P. Wohlfarth, D. Wagner
Letter

to the Editor

\section{References}

Sir,

I was interested to see that the EPS Advisory Committee on Publications has prepared a Europhysics Style Manual, the draft of which was published in Europhysics News vol. 3, no. 4, May 1972. While most of this appears useful and unobjectionable, there are two points on the presentation of references which I should like to raise as a physicist working as an information scientist.

Firstly, it is now accepted practice to give the final as well as the initial page number when referring to an article in a periodical. This practice indicates whether the item is a substantial article or a brief note, and it is very helpful in deciding whether to borrow the periodical or request a photocopy if it is not available in one's own library.

Secondly, it is much more useful if titles of articles are quoted as well as the periodicals in which they appear. Much waste of time and expense is often incurred in looking up and obtaining papers quoted in a list of references, only to find that the topic of interest is a very subsidiary part of their content. Now that authors are being encouraged to write informative titles, it seems only reasonable that these titles should be given to help readers judge the relevance of the papers quoted. The increased usefulness would easily outweigh the small additional space taken up.

The inclusion of titles and final page numbers is in accordance with the relevant ISO Recommendation ( $R 77$; Bibliographical references - essential elements, 1958) and the British Standard (BS 1629: 1950: Bibliographical references), and is the form illustrated by the Royal Society in its General Notes on the Preparation of Scientific Papers (2nd ed., 1965).

It would be a pity if EPS were to produce a style manual which leads authors and publishers to depart from these recommendations.
L.D. Will,
The City University,
St. John Street,
London ECIV 4PB.

Readers are invited to submit 'Letters to the Editor' either on items published in Europhysics News or on matters which would interest all European physicists. 